\title{
CLINICAL INTRODUCTION OF A NOVEL PROSTHESIS FOR VALVE-PRESERVING AORTIC ROOT RECONSTRUCTION FOR ANNULOAORTIC ECTASIA
}

Kenton J. Zehr, MDa

Mano J. Thubrikar, $\mathrm{PhD}^{\mathrm{b}}$

Geoffrey G. Gong, MD

J. Robert Headrick, MD

Francis Robicsek, MD, $\mathrm{PhD}^{\mathrm{b}}$
Objective: Most patients with annuloaortic ectasia are young. They are at risk for complications related to a lifetime of anticoagulation when composite grafts containing mechanical valves are used for reconstruction. The majority of patients have near normal valve cusps. Valve-preserving techniques have been developed to maintain valve function and avoid anticoagulation. The eddy currents occurring within the sinuses of Valsalva in the natural aortic root have been shown to be important in the smooth, gradual, and gentle closure of the valve. Compliance of the sinuses is important in reducing stress in the leaflets. A novel ascending aortic prosthesis with "built in" compliant sinuses (Robicsek-Thubrikar graft) was developed for clinical aortic root replacement.

Methods: Woven Dacron tubes were used to make the prostheses. Three precisely measured square pieces were cut to make the expandable, individual sinuses. Sewing the individual neo-sinuses to a scalloped end of the Dacron tube graft created the neo-sinotubular junction and sinotubular ridge. Five patients with annuloaortic ectasia underwent valve-preserving aortic root reconstruction.

Results: All intraoperative transesophageal echocardiographic images after the valve-preserving procedure showed a normal appearing root with $10 \%$ radial expansion of each sinus in systole. The space between the cusps and neo-sinus wall in systole was normal. No patient has more than mild aortic regurgitation.

Conclusions: Valve-preserving aortic root reconstruction with a novel Dacron prosthesis with compliant "built in" sinuses re-establishes normal aortic root geometry with near normal valve motion. This may enhance the durability of the valve-preserving operation. (J Thorac Cardiovasc Surg 2000;120:692-8)
$T_{\mathrm{cos}}^{\mathrm{h}}$ he Bentall procedure, which uses a mechanical valve conduit, has been the primary reconstructive technique for annuloaortic ectasia. ${ }^{1,2}$ In contrast, Yacoub and associates ${ }^{3}$ and David and Feindel ${ }^{4}$ have proposed

From the Division of Cardiovascular Surgery, Mayo Clinic, Rochester, Minn, ${ }^{\mathrm{a}}$ and the Heineman Medical Research Laboratory, Carolinas Medical Center, Charlotte, NC. ${ }^{\text {b }}$

Received for publication Feb 16, 2000; revisions requested May 30, 2000; revisions received June 19, 2000; accepted for publication June 26, 2000.

Address for reprints: Kenton J. Zehr, MD, Division of Cardiovascular Surgery, Mayo Clinic, 200 First St SW, Rochester, MN 55905 (E-mail: zehr.kenton@mayo.edu).

Copyright () 2000 by The American Association for Thoracic Surgery

$0022-5223 / 2000 \$ 12.00+0 \quad \mathbf{1 2 / 1 / 1 0 9 6 9 9}$

doi: $10.1067 / \mathrm{mtc} .2000 .109699$ valve-preserving aortic root reconstruction. Most patients requiring root reconstruction are young and have a connective tissue abnormality, for example, Marfan syndrome. They are at significant risk for complications related to a lifetime of anticoagulation. The source of the disease that results in dissection and rupture resides in the wall of the aorta. Despite evidence that the tissue within the valve cusps has abnormal fibrillin in patients with Marfan syndrome, the effect on functional integrity appears to be minor. Elastin crosslinking does not appear to play a prominent role in the more fibrous valve cusp. Intermediate-term success with valve-preserving root reconstruction has supported this theory.

The eddy currents occurring within the sinuses of Valsalva in the natural aortic root are considered to be 
important in the smooth, gradual, and gentle closure of the valve. ${ }^{5}$ On this basis, a design of an ascending aortic graft with "built in" sinuses was proposed in $1990 .^{6}$ Our recent studies have established that the compliance of the sinuses is perhaps even more important than the geometry for reducing stress in the leaflets and enhancing valve longevity. ${ }^{7}$ In accordance with this, we have now produced a novel Dacron prosthesis with compliant sinuses of Valsalva for aortic root replacement (Robicsek-Thubrikar graft). ${ }^{8}$ The purpose of this article is to present the first clinical implantations of this prosthesis, which re-establishes the geometric relationships of the aortic root.

\section{Methods \\ Clinical summaries}

PATIENT 1. A 29-year-old Native American woman had long-standing severe aortic regurgitation and a lupus-type syndrome. The root diameters were $22 \mathrm{~mm}$ at the anulus, 34 to $36 \mathrm{~mm}$ at the sinuses of Valsalva, and 36 to $38 \mathrm{~mm}$ at the sinotubular junction. The mechanism of regurgitation was a dilated sinotubular junction causing severely splayed-out commissures and lack of central coaptation of the cusps. She also had severely thickened left and noncoronary aortic cusps $(8-10 \mathrm{~mm})$ adjacent to the regurgitant jet. She was referred for a valve-sparing procedure because of her young age, reluctance to take warfarin sodium (Coumadin) because of the long-term risk of anticoagulation, and desire to have children.

PATIENT 2. A 47-year-old white man had familial Marfan syndrome and long-standing moderate aortic regurgitation. $\mathrm{He}$ also had polycystic kidney disease with a creatinine value of $1.6 \mathrm{mg} / \mathrm{dL}$. The root diameters were $27 \mathrm{~mm}$ at the anulus, $57 \mathrm{~mm}$ at the sinuses of Valsalva, and $55 \mathrm{~mm}$ at the sinotubular junction (Fig 1). The mechanism of aortic regurgitation was similar to that in patient 1 (Fig 1, B). The aortic cusps had some central thickening adjacent to the aortic regurgitation jet. The cusps were otherwise normal.

PATIENT 3. A 55-year-old white man had annuloaortic ectasia of unknown etiology. Dilation of the aortic root was discovered on a routine chest $\mathrm{x}$-ray film. He had no significant comorbidities. The root diameters were $26 \mathrm{~mm}$ at the anulus, $48 \mathrm{~mm}$ at the sinuses of Valsalva, and $46 \mathrm{~mm}$ at the sinotubular junction. He had significant anxiety related to the aneurysm and wished to avoid anticoagulation because of an active lifestyle.

PATIENT 4. A 73-year-old white woman had an acute type A dissection of a $60-\mathrm{mm}$ ascending aortic aneurysm involving the aortic root. There was extensive intramural hematoma and dissection extending deeply into the right and left coronary sinuses. She had severe aortic regurgitation. She required aortic arch replacement, oversewing of the right coronary artery ostial dissection, and a saphenous vein graft to the right coronary artery, in addition to the valve-preserving procedure.

PATIENT 5. A 48-year-old African American man had nonfamilial Marfan syndrome with bilateral subluxation of the lenses and severe scoliosis. He had severe aortic and mitral regurgitation and congestive heart failure. The root diameters were $25 \mathrm{~mm}$ at the anulus, $49 \mathrm{~mm}$ at the sinuses, and $45 \mathrm{~mm}$ at the sinotubular junction. He required additional bileaflet mitral valve repair and 3 coronary artery bypass grafts.

Prosthesis construction. A woven Dacron tube graft (Hemashield; Meadox Medicals, Inc, Oakland, NJ [subsidiary of Boston Scientific Corporation]) was chosen. A 24$\mathrm{mm}, 28-\mathrm{mm}, 26-\mathrm{mm}, 28-\mathrm{mm}$, and 28- $\mathrm{mm}$ tube was used for patients 1 to 5 , respectively. Three precisely measured square pieces were cut to anticipate individual sinuses measuring $95 \%$ of the diameter of the reconstructed root in height and two thirds of the diameter of the reconstructed root in width. Each square sinus piece started out with each side the length of the diameter of the tube. The $\mathrm{Z}$ folds in these pieces were turned $90^{\circ}$ compared with the folds of the tube graft to allow for lateral sinus expansion and to obtain the teardrop shape. A 4-0 polypropylene suture was used to create 2 purse-string sutures designed to form the expandable sinus (Fig 2, A) anticipating a 3-mm teardrop-shaped sinus bulge. The neosinotubular junction and sinotubular ridge were created by sewing the individually made sinuses to a scalloped end of the Dacron tube graft with the use of a fluted template and 40 polypropylene suture (Fig 2,B).

Surgical technique. The technique was similar for all patients. Cardiopulmonary bypass was established with venous drainage via a double-staged venous cannula in the right atrium and arterial return via the transverse aortic arch. The exception to this technique was cannulation of the right femoral artery in patient 4 . The left ventricle was vented via the right superior pulmonary vein and cold blood cardioplegia (antegrade and retrograde) was used for myocardial protection.

The ascending aorta was clamped and transected $2 \mathrm{~cm}$ proximal to the base of the innominate artery. Patient 4 required a period of hypothermic circulatory arrest for further aortic resection and arch replacement. The dilated root was opened and again transected $5 \mathrm{~mm}$ above the commissures. The left and right main coronary ostia were isolated on a Carrel patch. The sinuses were excised to $5 \mathrm{~mm}$ from the anulus. The commissures were suspended on stay sutures and the new sinotubular junction diameter was sized to allow central coaptation. Additionally, in patient 1 the chronically inflamed thickened tissue on the underside of the left and noncoronary cusps was shaved off with a 15-blade knife. This significantly increased cusp mobility.

Size 4-0 polypropylene sutures were used to suspend the commissures on the inside of the Dacron root prosthesis. The crown-shaped annular suture line was then sewn in place with a single-layer 4-0 polypropylene running suture. The continuous suture line incorporated the outer edge of the aortic anulus and the outer purse-string of each neo-sinus. This served to provide flexible annular fixation. The left and right coronary ostial buttons were reanastomosed to a 6- to 8-mm hole made in their respective neo-sinuses with a running 5-0 polypropylene suture. The prosthesis was measured to length and sewn to the distal ascending aorta with 4-0 polypropylene suture (Fig 3). 

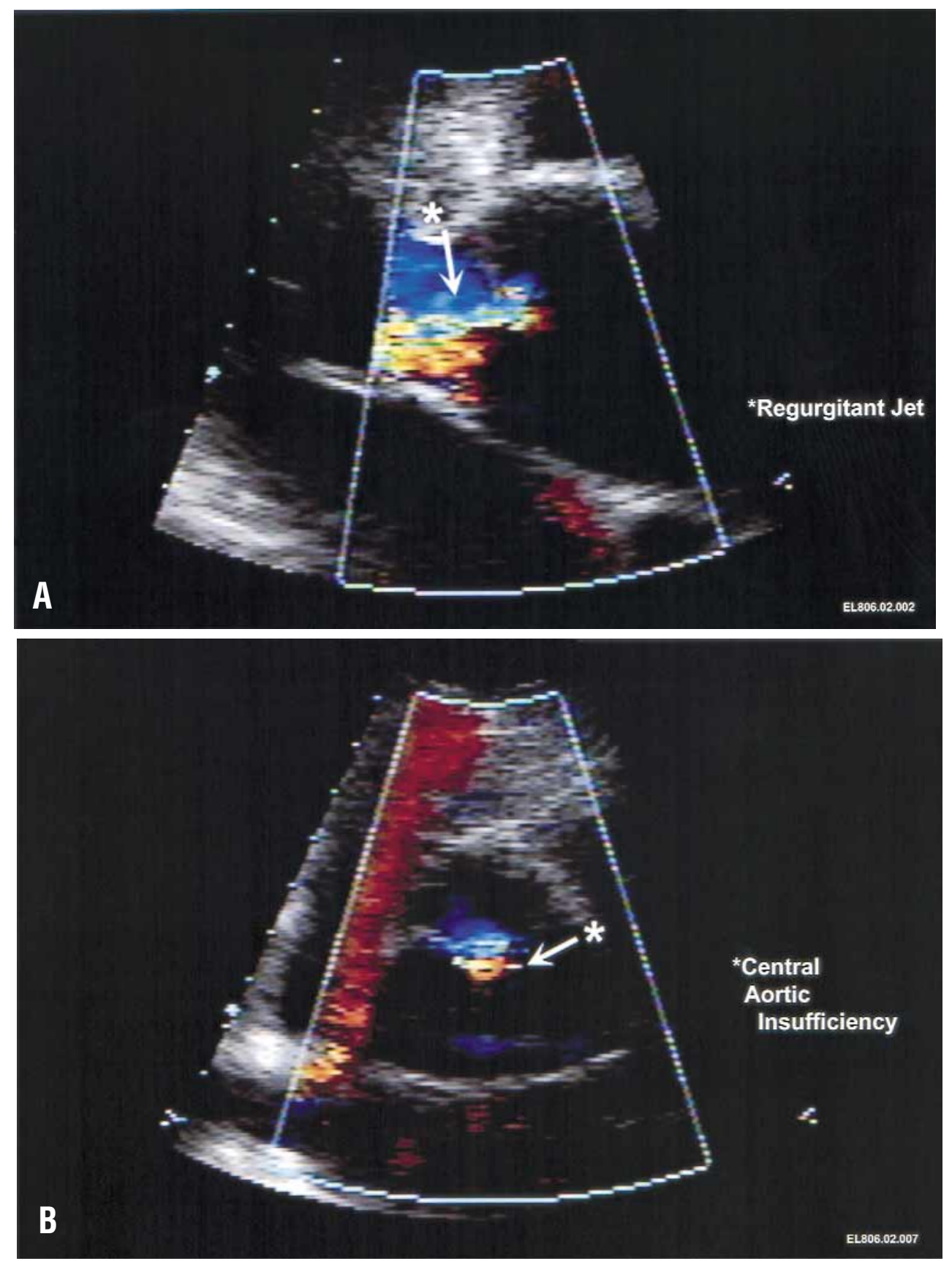

Fig 1. A, Preoperative transthoracic echocardiogram of patient 2 showing a long-axis view of the aortic valve, aortic root, and ascending aorta with the regurgitant jet. Note the aneurysmal dilatation at the sinus and sinotubular junction levels. B, Preoperative transthoracic echocardiogram of patient 2 showing a transverse view of the aortic cusps in diastole with poor central coaptation causing moderate aortic insufficiency.

\section{Results}

Intraoperative transesophageal echocardiographic images obtained after the valve-preserving procedure showed a normal appearing root with $10 \%$ radial expansion of each sinus in systole. The valve cusps coapted well above the anulus (Fig 4, A), and the space between the cusps and neo-sinus wall in systole was normal (Fig 4, B). Aortic regurgitation was mild. The arterial traces showed a crisp dicrotic notch. No murmurs were heard on auscultation. 


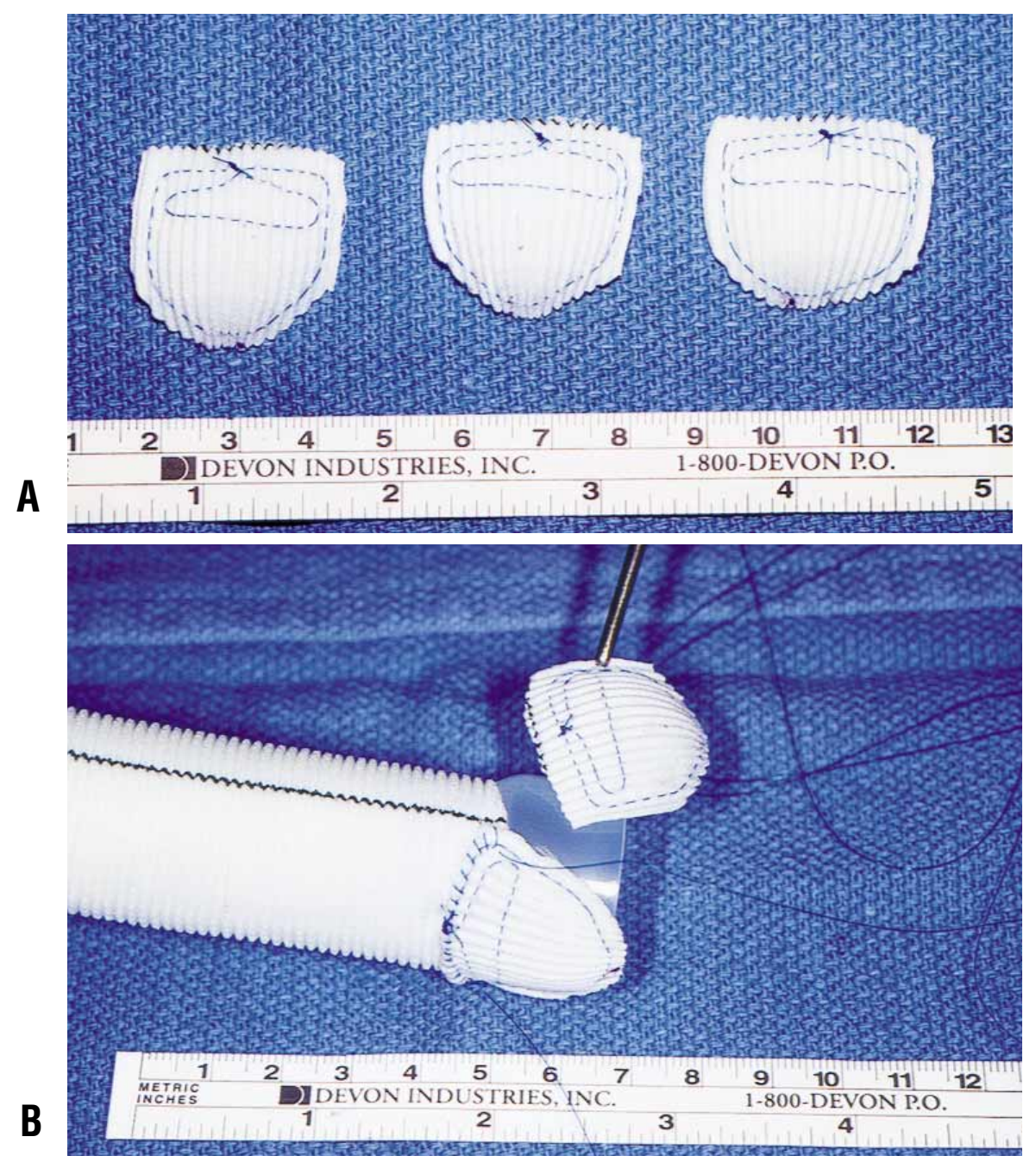

Fig 2. A, Three individual neo-sinuses, each with 2 purse-string sutures to allow for compliance as in the natural root. Note the orientation of the $\mathrm{Z}$ folds. $\mathbf{B}$, The sewing of the individual neo-sinuses to the neo-sinotubular junction.

\section{Comment}

Reconstruction of the aortic root affected by annuloaortic ectasia dates back to the early 1960s. The reconstructive techniques used were aortoplasty, tube graft replacement, and aortic valvuloplasty. Development of mechanical valves and synthetic tube conduits allowed replacement of the valve along with separate tubular graft replacement of the ascending aorta. Various techniques culminated in the Bentall procedure, which was introduced by Bentall and DeBono ${ }^{9}$ in 1968. They described the use of a composite graft, which incorporated the valve and a tubular conduit to replace the entire aortic root. Early experience was marred by significant complications of bleeding and pseudoaneurysm formation at suture lines. These problems were addressed by modifications including the introduction of albumin-impregnated vascular conduits, reattachments of the coronary ostia by a button technique, and felt buttressing of tenuous suture lines.

The safety and efficacy of the modified Bentall reconstruction have been documented by several large series. Gott and associates ${ }^{1}$ reported on 270 patients undergoing root replacement between 1976 and 1993: 252 Bentall composite graft repairs and 18 homograft root reconstructions. Of their patients, 187 had Marfan syndrome and another 53 had nonspecific medial degeneration. Thirty-day mortality was $4.8 \%$. Actuarial follow-up at 10 years was $73 \%$. Endocarditis developed 


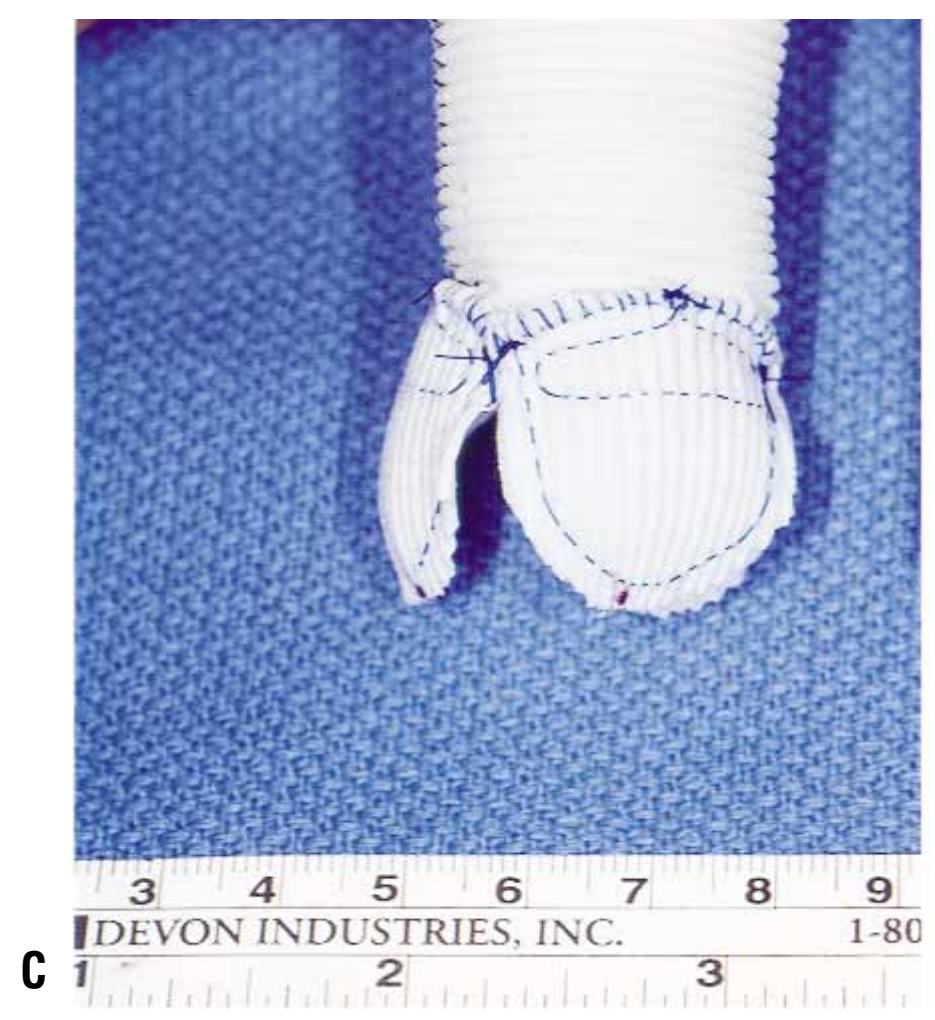

Fig 2. Cont'd. C, The completed tailored prosthesis.

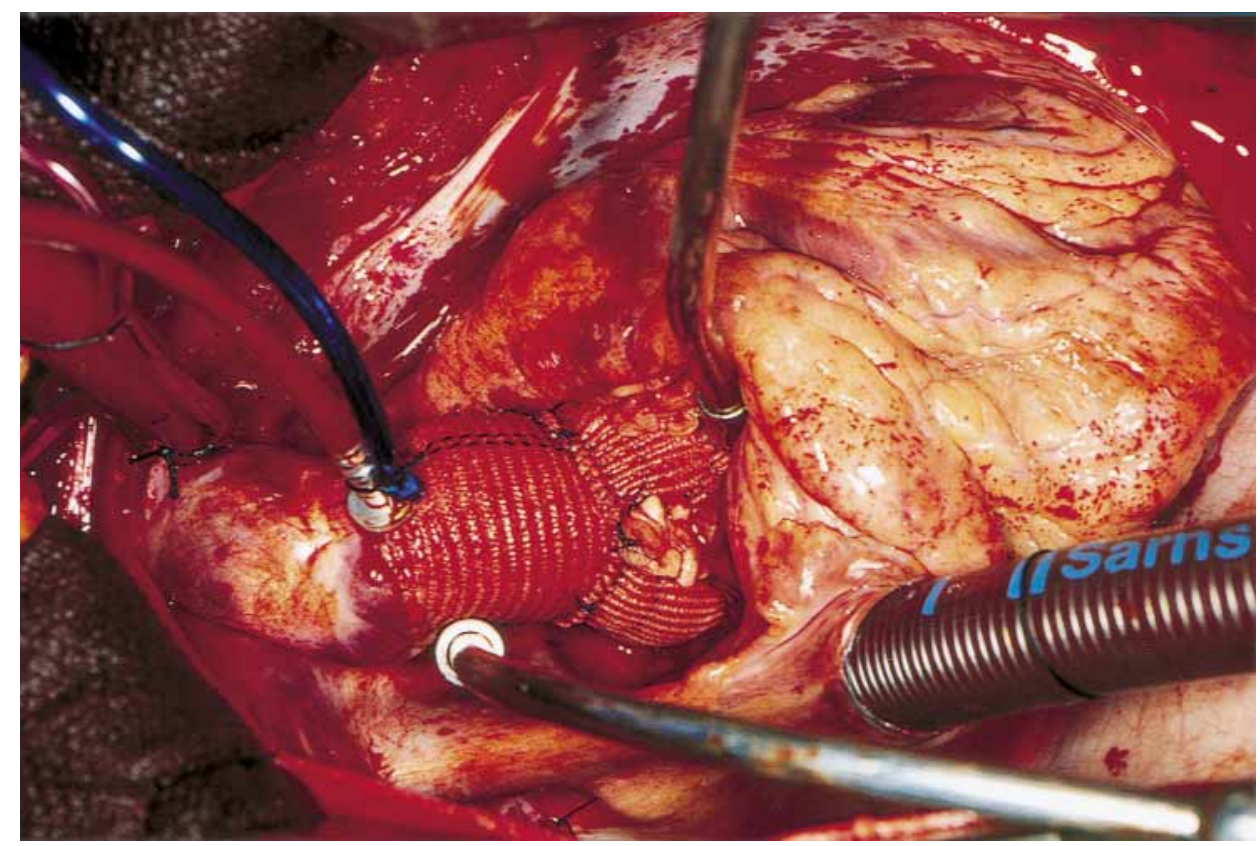

Fig 3. Intraoperative photograph showing the natural contour of the right and noncoronary sinuses after implantation. 

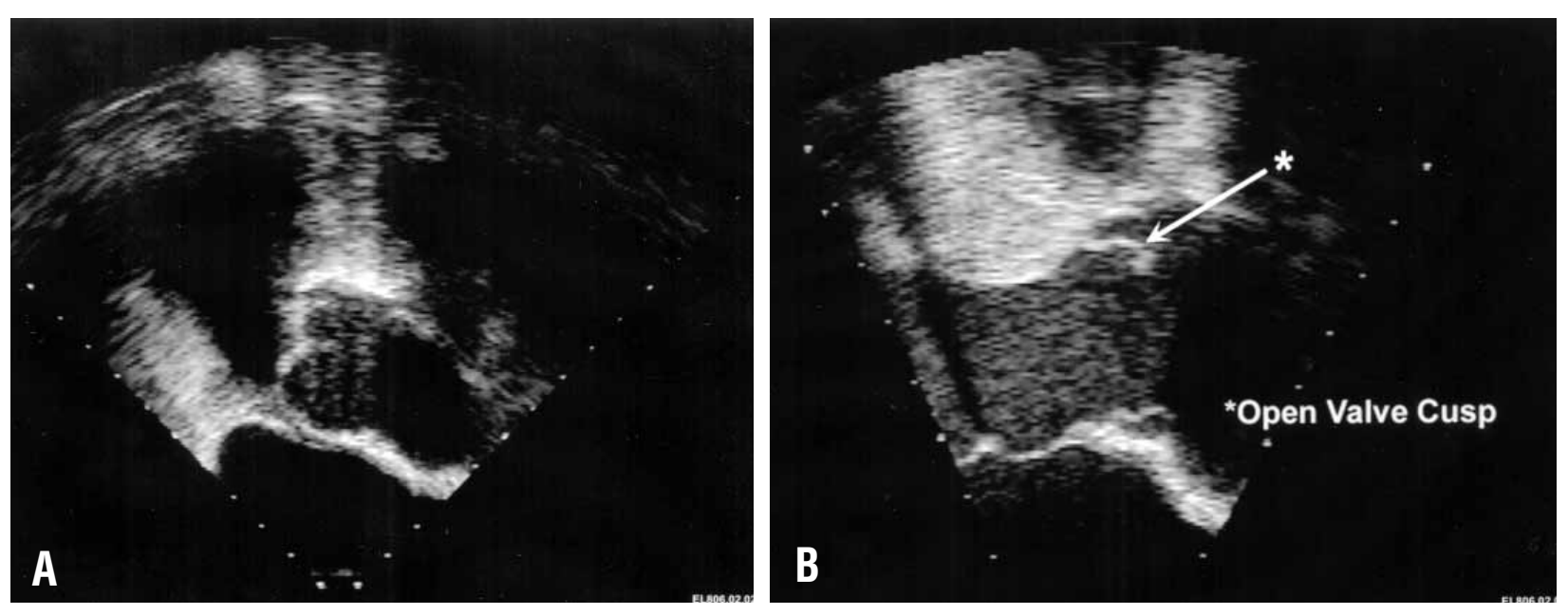

Fig 4. A, Intraoperative transesophageal echocardiographic image obtained after the valve-preserving procedure in patient 2 showing a long-axis view of the coapted valve cusps and neoaortic root in diastole. Note the normal level of coaptation above the annular level. B, Postoperative transthoracic echocardiogram of patient 2 showing a long-axis view of the cusps in systole. Note the position of the cusps suspended well away from the wall of the neo-sinuses.

in $14(5 \%)$ of 256 hospital survivors. Kouchoukos and colleagues $^{2}$ in 1991 presented a 16-year experience of 168 patients undergoing 172 aortic root replacements. Forty of these patients had Marfan syndrome. The hospital mortality rate for the overall series was $5 \%$. They concluded that use of the composite graft technique as a preferred method of treatment was valid. However, freedom from thromboembolism was only $82 \%$ at 12 years.

More recent advances have centered on valve-sparing procedures. The theoretic advantage is the avoidance of lifelong anticoagulation and the risks of thromboembolism, hemorrhage, endocarditis, and restricted hemodynamics. These procedures have a significant advantage for the young patient. Yacoub and coworkers ${ }^{3}$ reported a series of 168 patients who underwent a valve-sparing preservation technique, begun in 1979. Sixty-eight of these patients had Marfan syndrome. The technique involves replacement of the entire ascending aorta, sinotubular ridge, and sinuses of Valsalva with a Dacron tube graft cut into the mirror-image of the crown shape of the aortic anulus. Coronary ostia are reimplanted as buttons. The probabilities of reoperation were $3 \%, 11 \%$, and $11 \%$ at 1,5 , and 10 years, respectively. At follow-up, there was no or trivial aortic regurgitation in $63.6 \%$, mild to moderate in $33.3 \%$, and severe in $3 \%$. Another valve-sparing procedure was presented by David and Feindel ${ }^{4}$ in 1992. They described a technique in which the aortic root is reconstructed by resuspending the commissures and anulus within a Dacron tube graft. They reported on 102 patients, of whom 28 had the stigmata of Marfan syndrome. There were 2 operative deaths, 1 early failure necessitating composite replacement, and 1 failure at 2 years of follow-up. Five late deaths occurred. Six-year actuarial survival was $87 \%$. Importantly, no patients had thromboembolic or endocarditic complications. Only 3 patients had moderate aortic regurgitation at 3 to 108 months' follow-up.

These current techniques of valve-sparing aortic root reconstruction have limitations. In the David procedure, a Dacron tube graft is placed over the entire aortic root structure. This technique maintains valve competency by downsizing the sinotubular junction and allowing the aortic cusps to centrally coapt. The mechanism of failure is thought to be the result of the cusp abrading against the Dacron tube graft when the valve is opened. In addition, there are no sinuses of Valsalva, a fact that results in significantly abnormal stresses exerted on the cusp during opening and closing. ${ }^{10,11}$ The Yacoub technique ${ }^{3}$ uses a tube graft trimmed proximally to create 3 tongues that replace the expanded sinuses. This technique allows more normal cusp motion and lack of contact of the cusps with the Dacron. However, true sinuses are not created and the sinuses are not expanded significantly, which can result in significant cusp stress on opening and closing. $5,8,11$ This stress will reduce long-term durability of the valve cusps. An additional disadvantage of this technique might be the tendency of the Dacron tongues to splay 
out over time, resulting in progressive aortic insufficiency. As in Yacoub's technique, in our technique, a circumferential annuloplasty stitch, sinus-to-sinus commissuroplasty sutures, or an annuloplasty band from the left to the right coronary sinus could be incorporated in patients having connective tissue disorders or a dilated anulus. In patient 5 , three sinus-to-sinus commissuroplasty sutures were used for this purpose. Cochran and associates ${ }^{12}$ proposed a modification of tube graft reimplantation and scalloped remodeling to produce pseudosinuses. In their technique, the slightly scalloped tube graft was sewn in a subannular position. Resuspension of the commissures and slight graft plication caused by the annular suture line allow bulging in the sinus region. This takes advantage of the compliance of the Dacron tube. It does not create a tearshaped, natural sinus but does keep the Dacron away from the leaflet. The technique also stabilizes the anulus. This technique has been shown experimentally to result in significantly less stress and strain on the cusps compared with the Yacoub or David approaches. ${ }^{11}$

The aortic root is a complex 3-dimensional structure. The commissural attachments of the valve to the anulus represent the points of a 3-point crown from which the free margins of the valve cusps are suspended similar to the cables from the columns of a suspension bridge. The sinotubular junction is made up of the 3 commissural structures and the encircling, slightly thickened ridge of the aortic wall tissue at the junction of the sinuses of Valsalva and the ascending aorta. As the heart begins systole and pressure increases in the aortic root, the sinotubular junction expands $12 \%$. This helps to retract the free leaflet edges. Vortices are created within the sinuses of Valsalva and the cusps are suspended between these vortices and the outflowing blood within the aortic root. Thus, there is a net zero force from either side on the valve leaflet, which results in minimal stress on the tissue.

Central coaptation of the cusps requires that the diameter of the sinotubular junction nearly approximate the length of the free margin of each individual cusp. The diameter at the aorta at the sinotubular junction is $10 \%$ to $15 \%$ smaller than the diameter at the base of the anulus. The height of the leaflets is two thirds the length of the free margin of the leaflets.
Competency of the valve depends on maintenance of these geometric relations within the aortic root. ${ }^{8}$ There is often some variation of individual sinuses and distances between commissures. Our sinus sizing technique does not take into account these discrepancies. They can be adjusted for by suspension of the commissures at slightly different heights and by taking in more neo-sinus Dacron with the suture line in slightly smaller native sinuses.

The novel aortic root prosthesis recreates the geometric and functional aspects of natural aortic root and valve leaflets. We anticipate that its use will increase longevity of the spared valve cusps in these patients.

\section{REFERENCES}

1. Gott VL, Gillinov AM, Pyeritz RE, Cameron DE, Reitz BA, Greene PS, et al. Aortic root replacement: risk factor analysis of a seventeen-year experience with 270 patients. J Thorac Cardiovasc Surg 1995;109:536-44.

2. Kouchoukos NT, Wareing TH, Murphy SF, Perrillo JB. Sixteenyear experience with aortic root replacement: results of 172 operations. Ann Surg 1991;214:308-18.

3. Yacoub MH, Gehle P, Chandrasekaran V, Birks EJ, Child A, Radley-Smith R. Late results of valve-preserving operation in patients with aneurysms of the ascending aorta and root. J Thorac Cardiovasc Surg 1998;115:1080-90.

4. David TE, Feindel CM. An aortic valve-sparing operation for patients with aortic incompetence and aneurysm of the ascending aorta. J Thorac Cardiovasc Surg 1992;103:617-21.

5. Robicsek F, Thubrikar MJ. Role of sinus wall compliance in aortic leaflet function. Am J Cardiol 1999;84:944-6.

6. Robicsek F. Ascending aortic prosthesis: United States Patent Number 5139,15. August 18, 1992.

7. Thubrikar M. The aortic valve. Boca Raton (FL). CRC Press; 1990. p. 119-36.

8. Thubrikar MJ, Gong GG, Selim GA, Fowler B, Robicsek F. A new aortic root prosthesis with compliant sinuses for valve sparing operations. Circulation 1999;100(Suppl):I-461.

9. Bentall H, DeBono A. A technique for complete replacement of the ascending aorta. Thorax 1968;123:L338-9.

10. Leyh RG, Schmidtke C, Sievers HH, Yacoub MH. Opening and closing characteristics of the aortic valve after different types of valve-preserving surgery. Circulation 1999;100:2153-60.

11. Grande-Allen KJ, Cochran RP, Reinhall PG, Kunzelman KS. Recreation of sinuses is important for sparing the aortic valve: a finite element study. J Thorac Cardiovasc Surg 2000;119:753-63.

12. Cochran RP, Kunzelman KS, Eddy AC, Hofer BO, Verrier ED. Modified conduit preparation creates a pseudosinus in an aortic valve-sparing procedure for aneurysm of the descending aorta. $\mathrm{J}$ Thorac Cardiovasc Surg 1995;109:1049-58. 